\title{
Home confinement during the COVID-19: day-to-day associations of sleep quality with rumination, psychotic-like experiences and somatic symptoms
}

\author{
Péter Simor ${ }^{* 1,2}$, Polner $B^{*}{ }^{3}$, Báthori $N^{3}$, Sifuentes-Ortega $R^{2}$, Van Roy $A^{2}$ Albajara Sáenz $A^{2}$, \\ Luque González $\mathrm{A}^{4}$, Benkirane $\mathrm{O}^{2}$, Nagy $\mathrm{T}^{1}$, Peigneux $\mathrm{P}^{2}$.
}

1 Institute of Psychology, ELTE, Eötvös Loránd University, Budapest, Hungary.

2 UR2NF, Neuropsychology and Functional Neuroimaging Research Unit at CRCN - Center for Research in Cognition and Neurosciences and UNI - ULB Neurosciences Institute, Université Libre de Bruxelles (ULB), Brussels, Belgium.

3 Budapest University of Technology and Economics, Department of Cognitive Science

4 Universidad Autónoma de Madrid, Department of Biological and Health Psychology

Corresponding author:

Péter Simor, Ph.D.

Institute of Psychology, ELTE Eötvös Loránd University, Budapest, Hungary.

1064 Budapest, Izabella utca 46.

simor.peter@ppk.elte.hu

$+36302755968$

* The authors contributed equally to this work. 


\begin{abstract}
Due to the COVID-19 pandemic, populations from many countries have been confined at home for extended periods of time in stressful environmental and media conditions. Cross-sectional studies already evidenced deleterious psychological consequences, with poor sleep as a risk factor for impaired mental health. However, limitations of cross-sectional assessments are response bias tendencies, and the inability to track daily fluctuations in specific subjective experiences in extended confinement conditions. In a prospective study conducted across three European countries, we queried participants $(N=166)$ twice a day through an online interface about their sleep quality and their negative psychological experiences for two consecutive weeks. Focus was set on between-and within-person associations of subjective sleep quality with daytime experiences such as rumination, psychotic-like experiences, and somatic complaints about the typical symptoms of the coronavirus. Results show that daily reports of country-specific COVID19 deaths predicted increased negative mood, psychotic-like experiences and somatic complaints during the same day, and decreased subjective sleep quality the following night. Disrupted sleep was globally associated with negative psychological outcomes during the study period, and a relatively poorer night of sleep predicted increased rumination, psychotic-like experiences, and somatic complaints the following day. This temporal association was unidirectional since daytime reports of negative mental experiences were not associated with poor sleep quality on the following night. Our findings show that night-to-night changes in sleep quality predict how individuals cope the next day with daily challenges induced by home confinement.
\end{abstract}

\title{
Keywords
}

Coronavirus, sleep, rumination, somatic symptoms, psychotic-like, stress.

Funding The study has received funding from the European Union's Horizon 2020 research and innovation programme under the Marie Sklodowska-Curie grant agreement No 801505 at the Universite Libre de Bruxelles (ULB). The study was supported by the (Hungarian) National Research, Development and Innovation Office (NKFIH-1157-8/2019-DT, NKFI/OTKA K 128599 and NKFI FK 128100) and by the BMEBiotechnology FIKP grant of EMMI (BME FIKP-BIO).

\section{Disclosure Statement:}

The authors have no conflicts of interest to declare.

\section{Methodological statement:}

The methods were carried out in accordance with the relevant guidelines and regulations. 


\section{Acknowledgements}

The authors are grateful to Róbert Báthori for designing the user interface and the layout, and created the online platform for data collection.

\section{Abbreviations}

PTSD: Posttraumatic stress disorder

FDR: False discovery rate

PCL: PTSD Checklist

BDI: Beck Depression Inventory

PSQI: Pittsburgh Sleep Quality Index

O-LIFE: Oxford-Liverpool Inventory of Feelings and Experiences

GSQS: Groningen Sleep Quality Scale

PHQ: Patient Health Questionnaire 


\section{Introduction}

In December 2019 a new coronavirus (SARS-CoV-2) was identified as the pathogen of an acute respiratory syndrome reported first in Wuhan, China ${ }^{1}$. The World Health Organization announced the disease (eventually named COVID-19) as a public health emergency of international concern ${ }^{2}$, and in the next three months, the COVID-19 continued to spread all over the world rapidly growing into a global pandemic. In order to slow the propagation of the virus as well as to attenuate the burden on health care systems, many countries introduced unprecedented measures of home confinement requiring individuals to stay at home and limit outdoor activities to the most necessary purposes. The restrictions drastically changed many individuals' daily routines that within the menacing context of the pandemic could lead to severe mental health complaints ${ }^{3,4}$, urging health professionals to consider the psychological impact of COVID-195-7.

A number of cross-sectional surveys conducted amongst front-line medical staff ${ }^{8,9}$, university students ${ }^{10,11}$, and the general population ${ }^{12-14}$ corroborated the concerns regarding mental health by reporting a pronounced increase in anxiety, depression, and symptoms of posttraumatic stress disorder (PTSD). Beyond these warning signs of psychological distress, a great proportion of the respondents reported frequent sleep difficulties ${ }^{6,12,14-16}$ and poor sleep emerged as an important risk factor for mental health complaints ${ }^{3}$. Additionally, disrupted sleep mediated the link between threat perception (measured by the COVID-19 death count) and negative emotions in a longitudinal study ${ }^{17}$, and those who reported more sleep difficulties during than before the confinement exhibited higher levels of depression, anxiety, and stress ${ }^{18}$. These findings are in line with previous studies showing the critical impact of poor sleep on the development and maintenance of mental health complaints ${ }^{19-21}$, and the putative role of healthy sleep in emotional adaptation ${ }^{22-25}$. During the confinement, reduced physical activity and lower exposure to daylight, irregular sleep-wake schedules, difficulties following good sleep habits, excessive and anxietyprovoking media use, increased levels of stress and social isolation may have deleterious impact on sleep 
quality and render individuals more vulnerable to mental health problems ${ }^{26}$. Therefore, monitoring sleep complaints during the pandemic is particularly relevant from a mental health perspective.

Limitations of the above studies are that sleep quality and daytime symptoms were measured retrospectively (e.g. asking participants to rate sleep quality during the previous weeks or month) and data were collected using cross-sectional designs. Although the approach allows quick and economical assessments in large samples, it is extremely difficult in such designs to address the directionality of the associations (i.e., whether sleep disruption temporally predicts daytime dysfunctions or the other way around). In addition, retrospective questionnaires might be more prone to biases ${ }^{27,28}$, especially when individuals are queried in extremely unusual and stressful circumstances such as the COVID-19 pandemic. Studies indicate that retrospective self-reports are strongly biased by negative mood states $^{28}$, are subject to contamination between scales due to response bias tendencies ${ }^{29}$, and show little agreement with prospectively assessed measures of similar variables ${ }^{30}$. Furthermore, retrospective crosssectional studies can only account for differences between individuals and neglect intra-individual variability (i.e. moment-to-moment changes in sleep and mental complaints within the same individuals), whereas prospective assessments point to considerable within-subject variability in sleep quality ${ }^{31,32}$, daytime affective states ${ }^{33,34}$, and even in states associated with personality traits that are considered to be stable over time ${ }^{35}$.

To overcome these limitations, we conducted a two-week, prospective study investigating the associations between subjective sleep quality and daytime experiences during the confinement of the COVID-19 pandemic. Our prospective data collection allowed us to simultaneously examine associations across and within individuals. Hence, we tested whether individuals with poor sleep exhibited a higher level of mental health complaints during the two-week assessment period, and whether subtle night-tonight fluctuations in sleep quality were prognostic of increased negative experiences the next day (within 
individuals). To address the temporal directionality of these associations, we examined whether subtle changes in daytime reports predicted changes in subjective sleep quality the following night.

Widely used measures of anxiety, depression, and stress provide efficient means to estimate the severity of negative affect and the prevalence of psychopathological conditions ${ }^{3,13,15}$, but are not capable of capturing more specific and transient mental experiences that may impose an emotional burden on individuals during home confinement. Here, we focused on three factors particularly relevant within the context of the COVID-19 pandemic: rumination, psychotic-like experiences, and somatic complaints mimicking symptoms of COVID-19. Rumination refers to repetitive, intrusive, and hardly controllable thoughts about self-relevant situations and their underlying causes, and appears as a transdiagnostic factor in various psychopathological states ${ }^{36,37}$. Rumination exhibits transient, state-like variations in healthy individuals and is associated with neurophysiological indices of impaired emotional adaptation ${ }^{38}$. Psychotic-like experiences are unusual subjective experiences that phenomenologically resemble the symptoms of psychosis at a subclinical level (e.g., difficulties in controlling thoughts, cognitive anomalies, strange perceptions, and paranoid ideas), which are relatively common in the general population ${ }^{39}$. Psychotic-like experiences also exhibit considerable moment-to-moment fluctuations $\mathrm{s}^{40,41}$ and may increase under stressful circumstances ${ }^{40,42}$. Coronavirus anxiety (the fear of obtaining the virus) emerged as a novel mental health issue of severe concern ${ }^{43}$ that was associated with a number of psychological difficulties. Therefore, somatic complaints mimicking the most common symptoms of COVID-19 were examined as a measure of somatic symptom severity ${ }^{44}$ possibly related to the health anxiety provoked by the pandemic. Although day-to-day (intra-individual) fluctuations of somatic symptoms were mainly evidenced in clinical populations ${ }^{45,46}$, healthy individuals might also express pronounced variability in somatic symptoms in the context of the unprecedented coronavirus pandemic. We thus hypothesized that sleep disruptions would be associated with rumination, psychotic-like experiences, and somatic complaints during the study period, and that nights of poor sleep quality would eventually lead to worse 
daytime functioning the following day within an individual. Likewise, we examined the inverse direction, i.e., whether daytime rumination, psychotic-like experiences, and somatization could predict worse sleep quality the following night. Additionally, we reasoned that trait-like characteristics such as proneness for a dysregulated stress-response after exposure to a stressor (as reflected by PTSD-like symptoms ${ }^{47,48}$ ) and difficulties in controlling thoughts and attention (as reflected by cognitive disorganization ${ }^{49,50}$ ) would be predictive of increased maladaptation during the pandemic. Therefore, we examined the association of these retrospectively assessed psychopathological indices with daytime functioning over and above the hypothesized association with sleep.

\section{Results}

Screening of participants and cross-sectional measures. In the first phase of the study, participants from three European countries in which restrictive confinement measures were adopted (Belgium, Hungary, and Spain) responded to a survey including items addressing cognitive disorganization, posttraumatic stress symptoms, sleep quality, depression and general demographic data that served us to screen and select individuals for the ensuing prospective study (See Methods). Thirteen participants reported that they were tested positive for COVID-19, and their data were not considered in the subsequent analyses. The prevalence rate of clinically relevant (moderate) depression ${ }^{51}$ in our sample $(N=717,547$ females) was $23 \%$ (BE: $25.1 \%$, HU: $27 \%$, ESP: 18.4\%), and $19.2 \%$ of the respondents (BE: $21.1 \%, \mathrm{HU}: 25.6 \%$, ESP: $13.1 \%)$ scored over the cut-off score to identify clinically relevant symptoms of PTSD ${ }^{52}$ corroborating previous reports about the substantial increase of mental health problems during the pandemic ${ }^{3}$. Although moderate differences emerged across the countries with respect to PTSD symptoms, schooland work related stress, and cognitive disorganization (See Supplementary Materials), the psychometric measures of mental health complaints exhibited similar patterns of correlations in our subsamples (Figure 1). 


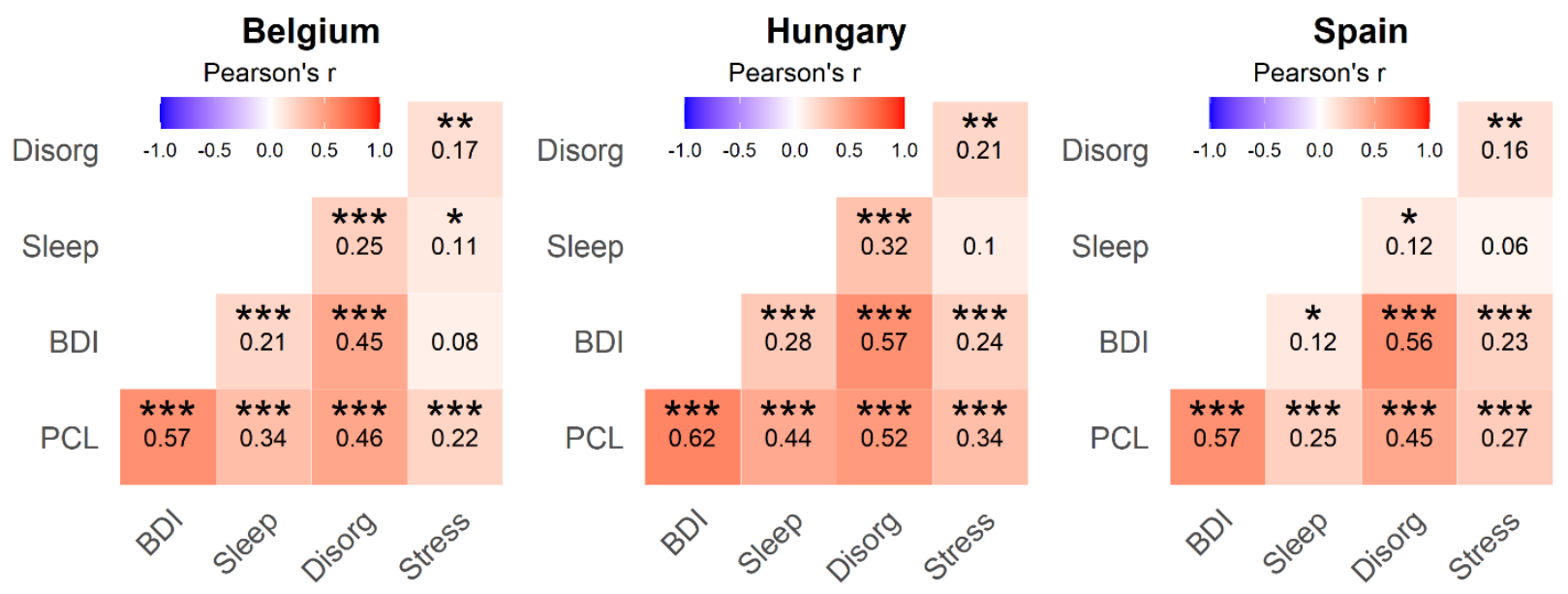

Figure 1. Cross-sectional survey. Pearson's correlation coefficients between the assessed variables among the Belgian, Hungarian, and Spanish sample of respondents $(N=717)$. The colors and numbers indicate Pearson's r-values, asterisks correspond to False Discovery Rate (FDR) corrected p-values (*** $p<0.001$, $\left.{ }^{* *} p<0.01,{ }^{* *} p<0.05\right)$. Stress: the increase of school-and job-related stress due to the confinement; Disorg: Cognitive Disorganization; Sleep: subjective sleep disturbances; BDI: Beck Depression Inventory; PCL: Posttraumatic stress disorder checklist.

Prospective study: Adherence to daily questionnaires. 184 individuals (146 (79 \%) females, age 18-69 years, $M_{\text {age }}=26.28, S D=7.42$ ) were assigned for the second phase of the study assessing daily questionnaires during two-weeks. $22 \%$ of the respondents completed all questionnaires, and $51 \%$ completed more than $80 \%$ of the daily questionnaires. Nine percent of the participants responded to less than $50 \%$ of the morning or the evening questionnaires, and their data were excluded from further analyses yielding to the prospective data of 166 individuals. 


\section{COVID-19-related deaths and days since the introduction of confinement predict daytime}

functioning. The first analyses of the prospective study focused on the day-to-day associations of mental health indices with the perceived context of the confinement. COVID-19-related deaths and the time spent in confinement were the objective measures reflecting the circumstances of the pandemic during the study period. We explored whether daily reports of mental health indices were associated with the daily numbers of COVID-19-related deaths worldwide and in the country of the respondents. Moreover, since the number of deaths had not been independent of the time spent since the introduction of the restrictive measures, we also examined the influence of time (expressed as a fraction of weeks) spent in confinement on daily reports of mood, rumination, psychoticlike experiences, somatic complaints, sleep disruption, and sleep duration. The number of COVID-19related deaths in the country predicted more negative mood $(b=-0.009, \mathrm{Cl}:-0.018--0.001)$, psychotic-like experiences $(b=0.004, \mathrm{Cl}: 0.002-0.006)$, and somatic complaints during the day $(b=0.005, \mathrm{Cl}: 0.003-0.007)$, and worse subjective sleep quality during the following night $(b=$ $0.003, \mathrm{Cl}: 0.001-0.005)$, whereas the number of worldwide deaths predicted more somatic complaints during the day $(\mathrm{b}=0.020, \mathrm{Cl}$ : $0.009-0.030)$ and prolonged sleep duration the following night $(\mathrm{b}=0.054, \mathrm{Cl}: 0.009-0.099)$. The number of weeks since the introduction of confinement in the country had a negative linear and a positive quadratic effect on rumination (linear $b=-0.333, \mathrm{Cl}:-0.432--0.234$, quadratic $\mathrm{b}=0.023, \mathrm{Cl}: 0.014-0.032$ ), psychotic-like experiences (linear $\mathrm{b}=-0.376, \mathrm{Cl}:-0.483--0.269$, quadratic $\mathrm{b}=0.024, \mathrm{Cl}: 0.015-0.034$ ) and somatic complaints (linear $b=-0.124, \mathrm{Cl}:-0.210--0.037$, quadratic $b=0.009, \mathrm{Cl}: 0.001-0.016$ )

(See Table 1). Further inspection of the plots of predicted values suggested that there was an 
overall improvement of daytime functioning with time spent in confinement. Moreover, the steepness of the curve (reflecting the gradual amelioration of daily symtpoms) was decreasing with time, indicating that the amelioration of mental health exhibited a slowing trend. These findings indicate that day-to-day fluctuations in mental health conditions were specifically associated with objective measures (number of deaths and days spent in confinement) reflecting the context of the confinement.

\begin{tabular}{|c|c|c|c|c|c|c|c|c|c|c|c|c|}
\hline \multirow[b]{2}{*}{ Predictors } & \multicolumn{2}{|c|}{ Positive mood } & \multicolumn{2}{|c|}{ Rumination } & \multicolumn{2}{|c|}{ Psychotic-like experiences } & \multicolumn{2}{|c|}{ Somatic complaints } & \multicolumn{2}{|c|}{ Sleep disruption } & \multicolumn{2}{|c|}{ Sleep duration } \\
\hline & Estimates & $95 \% \mathrm{CI}$ & Estimates & $95 \% C I$ & Estimates & $95 \% \mathrm{CI}$ & Estimates & $95 \% \mathrm{CI}$ & Estimates & $95 \% C I$ & Estimates & $95 \% C I$ \\
\hline Intercept & $4.881^{* * *}$ & $3.422-6.339$ & $1.101^{* * *}$ & $0.768-1.434$ & $1.265^{* * *}$ & $0.892-1.638$ & $0.477^{* *}$ & $0.176-0.779$ & $0.439^{*}$ & $0.096-0.782$ & $7.672^{* * *}$ & $6.550-8.795$ \\
\hline Age & -0.000 & $-0.019-0.019$ & $0.005^{*}$ & $0.000-0.010$ & 0.003 & $-0.003-0.010$ & -0.001 & $-0.006-0.004$ & 0.004 & $-0.000-0.008$ & $-0.027^{* * *}$ & $-0.041--0.014$ \\
\hline Gender (male) & -0.180 & $-0.487-0.127$ & $-0.098^{*}$ & $-0.181--0.016$ & -0.052 & $-0.156-0.052$ & $-0.146^{* * *}$ & $-0.229--0.063$ & $-0.077^{*}$ & $-0.148--0.006$ & $-0.410^{* * *}$ & $-0.630--0.190$ \\
\hline PTSD-like symptoms & 0.000 & $-0.011-0.012$ & $0.008^{* * *}$ & $0.005-0.011$ & $0.011^{* * *}$ & $0.007-0.015$ & $0.008^{* * *}$ & $0.004-0.011$ & $0.003^{*}$ & $0.001-0.006$ & 0.004 & $-0.004-0.013$ \\
\hline Cognitive Disorganisation & $-0.116^{* * *}$ & $-0.167--0.065$ & $0.023^{* *}$ & $0.009-0.037$ & $0.037^{* * *}$ & $0.019-0.054$ & $0.032^{* * *}$ & $0.018-0.045$ & $0.020^{* *}$ & $0.008-0.032$ & -0.034 & $-0.070-0.003$ \\
\hline Deaths related to COVID-19 (in country, 10/day) & $-0.009^{*}$ & $-0.018--0.001$ & 0.001 & $-0.001-0.003$ & $0.004^{* *}$ & $0.002-0.006$ & $0.005^{* * *}$ & $0.003-0.007$ & $0.003^{* *}$ & $0.001-0.005$ & 0.004 & $-0.003-0.010$ \\
\hline Deaths related to COVID-19 (global, 1000/day) & -0.049 & $-0.107-0.010$ & 0.011 & $-0.002-0.024$ & 0.008 & $-0.005-0.022$ & $0.020^{* * *}$ & $0.009-0.030$ & -0.008 & $-0.021-0.005$ & $0.054^{*}$ & $0.009-0.099$ \\
\hline Weeks spent in confinement (linear) & 0.263 & $-0.183-0.710$ & $-0.333^{* * *}$ & $-0.432--0.234$ & $-0.376^{* * *}$ & $-0.483--0.269$ & $-0.124^{* *}$ & $-0.210--0.037$ & -0.058 & $-0.164-0.049$ & 0.316 & $-0.034-0.665$ \\
\hline Weeks spent in confinement (quadratic) & -0.024 & $-0.063-0.015$ & $0.023^{* * *}$ & $0.014-0.032$ & $0.024^{* * *}$ & $0.015-0.034$ & $0.009^{*}$ & $0.001-0.016$ & 0.004 & $-0.005-0.013$ & -0.026 & $-0.056-0.004$ \\
\hline \multicolumn{13}{|l|}{ Random Effects } \\
\hline$\sigma^{2}$ & 1.7040 & & 0.0805 & & 0.0917 & & 0.0559 & & 0.0924 & & 1.0179 & \\
\hline$\tau_{00}$ & $0.5221_{\text {id }}$ & & $0.0411_{\text {id }}$ & & 0.0695 id & & 0.0414 id & & 0.0284 id & & 0.2577 id & \\
\hline ICC & 0.2345 & & 0.3380 & & 0.4314 & & 0.4253 & & 0.2348 & & 0.2020 & \\
\hline $\mathrm{N}$ & $166_{\text {id }}$ & & $166_{\text {id }}$ & & $166_{\text {id }}$ & & $158_{\text {id }}$ & & $166_{\text {id }}$ & & 166 id & \\
\hline Observations & 1984 & & 1984 & & 1984 & & 1893 & & 2011 & & 2010 & \\
\hline Marginal $\mathrm{R}^{2} /$ Conditional $\mathrm{R}^{2}$ & $0.053 / 0.27$ & & $0.175 / 0.454$ & & $0.245 / 0.57$ & & $0.252 / 0.57$ & & $0.062 / 0$ & & $0.056 / 0.24$ & \\
\hline
\end{tabular}

Table 1. The association of the number of deaths related to COVID-19 and of the days spent in confinement with daily indices of mental health, beyond the effects of age, gender, PTSD-like symptoms and cognitive disorganization. Daily reports of COVID-19 related deaths were associated with mood, psychotic-like experiences, and somatic complaints during the day, and worse subjective sleep quality the following night. The number of global deaths predicted more somatic complaints during the day and prolonged sleep duration the following night. The time spent in confinement was inversely related to the reported levels of rumination, psychotic-like experiences, and somatic complaints. $\sigma 2$ : residual variance; t00: variance of random intercept; $\tau 11$ : variance of random slope; $\rho 01$ : correlation between random intercept and slope; ICC: intraclass correlation; N: number of participants. Marginal R2: variance explained by fixed effects; Conditional $\mathrm{R}_{2}$ : variance explained by fixed and random effects. P-values were computed with Satterthwaite's approximation. 

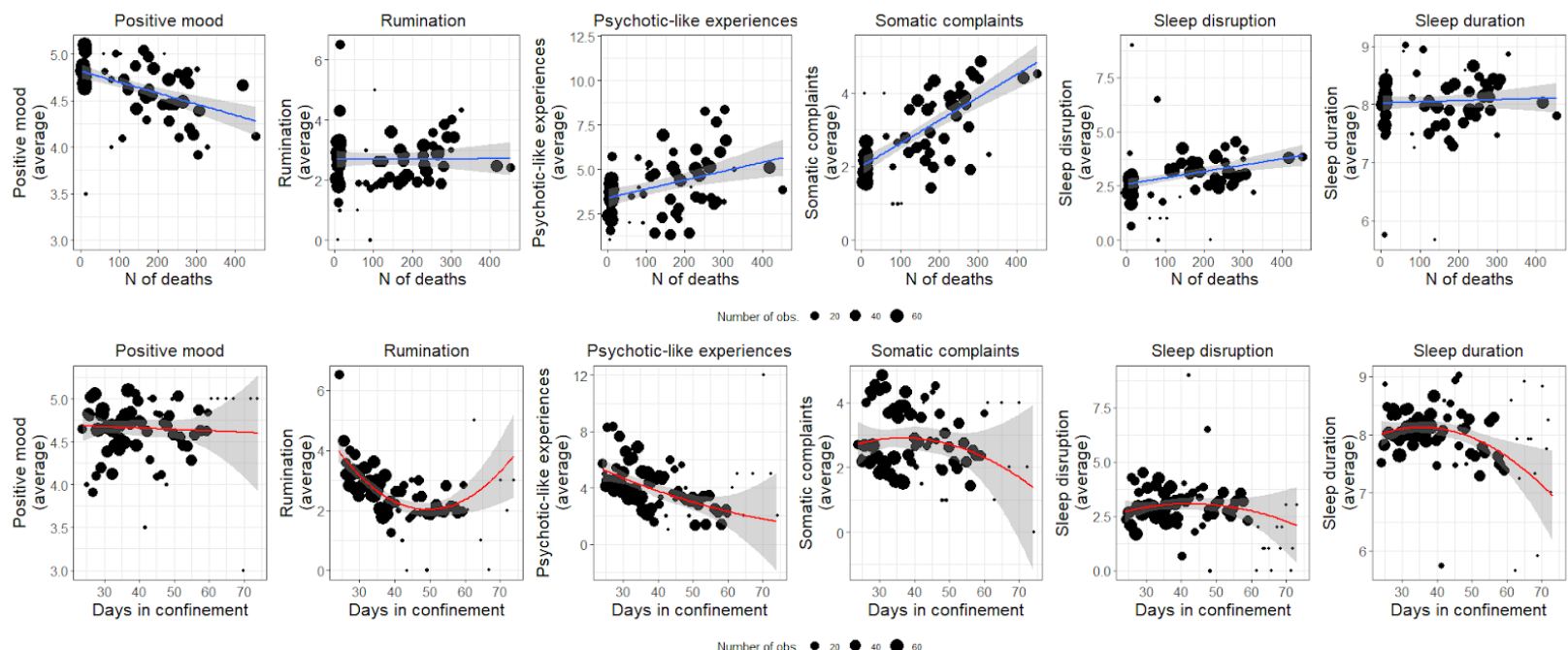
Top row: $x$-axis shows number of deaths related to COVID-19 at the country level
Bottom row: $x$-axis shows number of days since the introduction of the confinement

Figure 2. Mental health in the context of the confinement. Associations between the number of COVID19-related deaths at the country level (linear, shown on the $x$-axis in figures in the top row) and days since the introduction of confinement (quadratic, shown on the x-axis in figures in the bottom row) with daytime functioning, sleep disruption, and sleep quality. Daily reports of COVID-19 deaths were predictive of increased negative mood, more psychotic-like experiences, and somatic complaints during the day, and worse subjective sleep quality the following night. Daily reports of rumination, psychotic-like experiences, and somatic symptoms decreased as time elapsed since the introduction of the confinement, but we also observed a quadratic effect indicating that the amelioration of mental health exhibited a slowing trend. In the plots, each point represents the average of the given measure for a day within a country and the trend lines are weighted for the number of observations for the given day in a country. Shades show $95 \%$ confidence intervals. Note that confidence intervals become wider due to a lower number of observations around later time points after the introduction of the confinement.

Night-to-night fluctuations in sleep quality predict daytime functioning. We examined if subjective sleep disruption (on average) was associated with increased rumination over the two-week study period, and whether night-to-night variations in sleep quality were associated with changes in daily rumination within individuals. Subjective sleep quality was associated with rumination in both cases: more disrupted sleep (on average) was associated with increased rumination over the two-week study period $(\mathrm{b}=0.027, \mathrm{Cl}$ : $0.006-0.047, p<0.05$ ), and a relatively poorer night of sleep (i.e. reduction of sleep quality compared to the individual, two-week average) predicted relatively increased rumination the following day $(b=0.010$, $\mathrm{Cl}: 0.005-0.016, \mathrm{p}<0.001)$. In addition, trait-like, retrospective measures of posttraumatic symptoms 
(assessed by the PCL-5), and cognitive disorganization were both predictive of increased rumination over the study period (PCL-5: $b=0.007, \mathrm{Cl}: 0.004-0.010, p<0.001$, Cognitive Disorganization: $b=0.019, \mathrm{Cl}$ : $0.006-0.033, p=0.006$ ). Figure $3 /$ A illustrates the association between sleep disruption and rumination between- and within individuals, and statistical model parameters are specified in Table 2.

Next, we performed a similar analysis regressing the same variables on psychotic-like experiences. As expected, worse sleep quality was globally associated with increased reports of psychotic-like experiences $(b=0.054, \mathrm{Cl}: 0.029-0.079, \mathrm{p}<0.001)$. Moreover, night-to-night changes in sleep quality within individuals were also linked to increased psychotic-like experiences the following day, that is, worse sleep quality predicted more psychotic-like experiences the next day $(b=0.010, \mathrm{Cl}: 0.004-0.016, p<0.01)$. PTSD-like symptoms (PCL-5: $b=0.009, \mathrm{Cl}: 0.006-0.013, \mathrm{p}<0.001)$ and cognitive disorganization $(\mathrm{b}=$ $0.03, \mathrm{Cl}: 0.013-0.047, \mathrm{p}<0.001$ ) were both predictive of increased psychotic-like experiences (see Figure 3/B and Table 2).

Likewise, we investigated the links between sleep disruption and the daily experience of somatic symptoms of the COVID-19. At the between-subject level, subjective sleep disruption was positively associated with reports of somatic symptoms: the within-person average of sleep disruption was associated with a higher rate of somatic complaints over the course of the study $(\mathrm{b}=0.056, \mathrm{Cl}$ : $0.036-0.076, p<0.001)$. Regarding daily fluctuations within individuals, relatively worse sleep quality on a given night was associated with more severe somatic symptoms the following day $(b=0.007, \mathrm{Cl}$ : $0.002-0.012, \mathrm{p}<0.01)$. PCL-5 scores (PCL-5: $\mathrm{b}=0.005, \mathrm{Cl}: 0.002-0.009, \mathrm{p}<0.001)$ and cognitive disorganization $(b=0.026, \mathrm{Cl}: 0.013-0.039, p<0.001$ ) both emerged as significant predictors of (increased) somatic symptoms in the statistical model (see Figure 3/C and Table 2).

In sum, disrupted sleep was associated with increased rumination, psychotic-like experiences, and somatic symptom severity as measured prospectively during the two-week study period. Moreover, night- 
to-night variations in subjective sleep quality predicted next-day's functioning, indicating that a relatively worse night of sleep was more likely to lead to increased rumination, psychotic-like experiences, and somatic symptoms the following day.
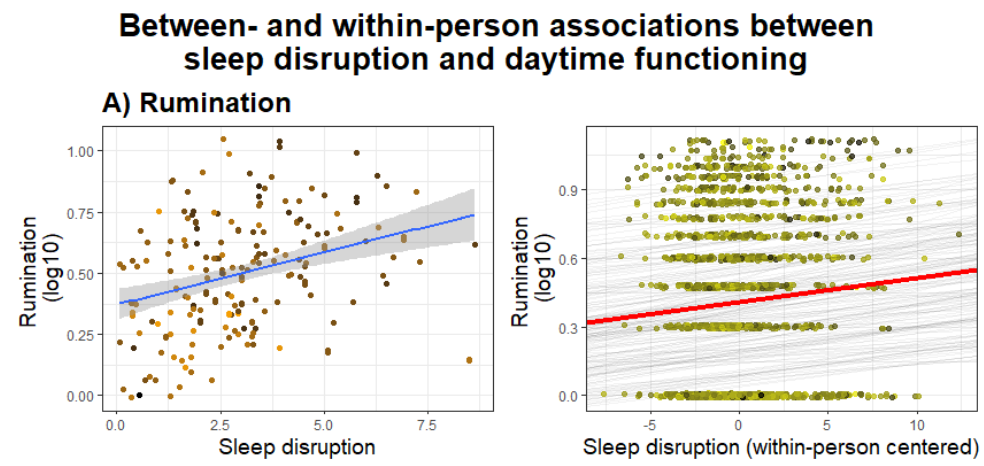

B) Psychotic-like experiences
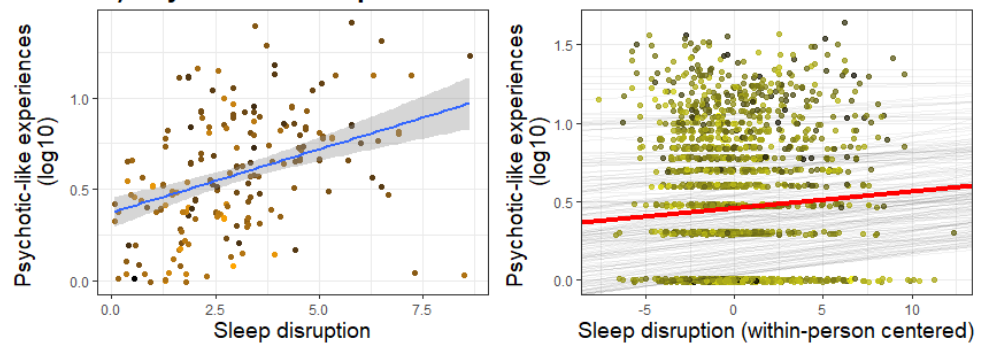

C) Somatic complaints
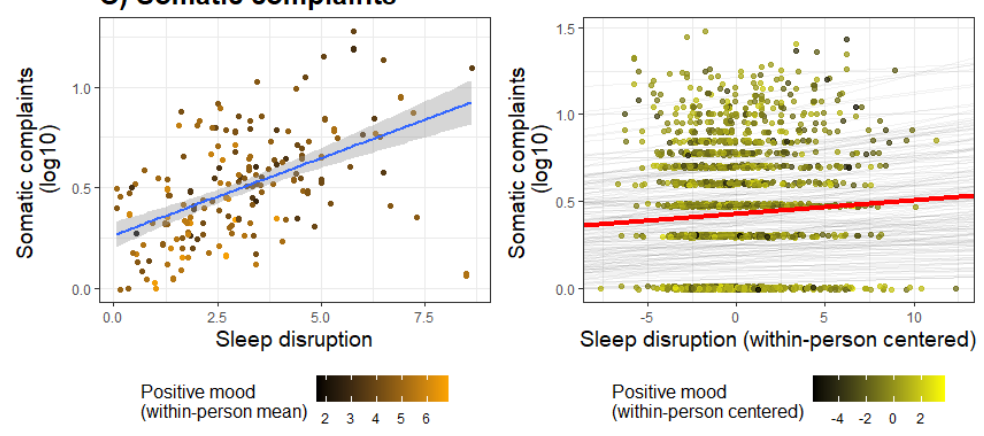

Figure 3. Prospective data analyses. Left column: Individual differences in subjective sleep disruption are associated with increased rumination, psychotic-like experiences, and somatic complaints during the twoweek study period. The scatterplots of the left column represent the two-week averages of sleep disruption (x-axes) and daytime experiences ( $y$-axes). Points with brighter and darker colors indicate positive and negative ratings of mood, respectively. Note that the brighter colored points (reflecting more positive mood) are more prominent in case of lower rumination, psychotic-like experiences, and somatic 
symptoms. Right column: Day-to-day associations between subjective sleep disruption and next day's rumination, psychotic-like experiences, and somatic complaints. Subjective sleep disruption as compared to the individual two-weekly averages, that is, relatively worse nights of sleep during the study period are associated with increased rumination, psychotic-like experiences, and somatic complaints the following day. Grey lines indicate the regression slopes of each individual fitted to their day-to-day reports of sleep quality and daytime experiences (individual means and slopes). The red lines show the regression slopes fitted to all data points (overall mean and slope). Points with brighter and darker colors indicate positive and negative ratings of mood respectively. As indicated by the color of the data points, more positive daily mood is associated with lower rumination, psychotic-like experiences, and somatic symptoms (See Table 2).

Associations between sleep disruption and subsequent daytime functioning

\begin{tabular}{|c|c|c|c|c|c|c|}
\hline \multirow[b]{2}{*}{ Predictors } & \multicolumn{2}{|c|}{ Rumination } & \multicolumn{2}{|c|}{ Psychotic-like experiences } & \multicolumn{2}{|c|}{ Somatic complaints } \\
\hline & Estimates & $95 \% C I$ & Estimates & $95 \% \mathrm{CI}$ & Estimates & $95 \% C I$ \\
\hline Intercept & $0.164^{*}$ & $0.014-0.313$ & 0.125 & $-0.056-0.306$ & $0.166^{*}$ & $0.018-0.314$ \\
\hline Age & 0.000 & $-0.004-0.005$ & -0.003 & $-0.009-0.003$ & -0.003 & $-0.007-0.002$ \\
\hline Gender (male) & -0.080 & $-0.162-0.002$ & -0.001 & $-0.100-0.098$ & $-0.099^{*}$ & $-0.179--0.019$ \\
\hline PTSD-like symptoms & $0.007^{* * *}$ & $0.004-0.010$ & $0.009^{* * *}$ & $0.006-0.013$ & $0.005^{* * * *}$ & $0.002-0.009$ \\
\hline Cognitive Disorganisation & $0.019^{* *}$ & $0.006-0.033$ & $0.030^{* * *}$ & $0.013-0.047$ & $0.026^{* * *}$ & $0.013-0.039$ \\
\hline Sleep disruption (within-person mean) & $0.027^{*}$ & $0.006-0.047$ & $0.054^{* * *}$ & $0.029-0.079$ & $0.056^{* * *}$ & $0.036-0.076$ \\
\hline Sleep disruption (within-person centered) & $0.010^{* * *}$ & $0.005-0.016$ & $0.010^{* *}$ & $0.004-0.016$ & $0.007^{* *}$ & $0.002-0.012$ \\
\hline \multicolumn{7}{|l|}{ Random Effects } \\
\hline$\sigma^{2}$ & \multicolumn{2}{|l|}{0.0835} & \multicolumn{2}{|l|}{0.0963} & \multicolumn{2}{|l|}{0.0567} \\
\hline$\tau_{00}$ & \multicolumn{2}{|l|}{0.0383 id } & \multicolumn{2}{|l|}{$0.0595 \mathrm{id}$} & \multicolumn{2}{|l|}{0.0363 id } \\
\hline$\tau_{11}$ & \multicolumn{2}{|c|}{0.0002 id.gsqs_sum_centered } & \multicolumn{2}{|c|}{0.0002 id.gsqs_sum_centered } & \multicolumn{2}{|c|}{0.0002 id.gsqs_sum_centered } \\
\hline$\rho_{01}$ & \multicolumn{2}{|l|}{0.1754 id } & \multicolumn{2}{|l|}{-0.3809 id } & \multicolumn{2}{|l|}{$0.2269 \mathrm{id}$} \\
\hline ICC & \multicolumn{2}{|l|}{0.3208} & \multicolumn{2}{|l|}{0.3884} & \multicolumn{2}{|l|}{0.4003} \\
\hline $\mathrm{N}$ & \multicolumn{2}{|l|}{166 id } & \multicolumn{2}{|l|}{166 id } & \multicolumn{2}{|l|}{158 id } \\
\hline Observations & \multicolumn{2}{|l|}{1884} & \multicolumn{2}{|l|}{1884} & \multicolumn{2}{|l|}{1800} \\
\hline Marginal $\mathrm{R}^{2}$ / Conditional $\mathrm{R}^{2}$ & \multicolumn{2}{|c|}{$0.142 / 0.417$} & \multicolumn{2}{|c|}{$0.232 / 0.530$} & \multicolumn{2}{|c|}{$0.268 / 0.561$} \\
\hline
\end{tabular}

Table 2. Summary of mixed models examining associations between sleep quality and subsequent daytime functioning. $\sigma 2$ : residual variance; 000 : variance of random intercept; $\tau 11$ : variance of random slope; 01 : correlation between random intercept and slope; ICC: intraclass correlation; $\mathrm{N}$ : number of participants. Marginal R2: variance explained by fixed effects; Conditional $R^{2}$ : variance explained by fixed and random effects. P-values were computed with Satterthwaite's approximation. 
Subjective sleep quality predicts next day functioning regardless of mood fluctuations. Sleep disruption was linked to negative mood based on the averages of the study period (between-individuals; $b=-0.1$, $\mathrm{Cl}:-0.18--0.02, \mathrm{p}=0.01$ ), and daily changes in sleep disruption were temporally associated with more negative mood the next-day (within-individuals; $b=-0.05, \mathrm{Cl}:-0.07--0.02, p<0.001$ ). To rule out that the associations of sleep disruption with rumination, psychotic-like experiences, and somatic symptoms (between-and within individuals) were not merely explained by the covariance of sleep disruption with negative mood, we entered the variable mood as an additional predictor into our models focusing on the links between sleep quality and daytime functioning. Although negative mood was associated with increased rumination, psychotic-like experiences, and somatic symptoms on both the between- and within-individual levels, the two-week averages of, as well as the daily variations in subjective sleep disruption remained significant predictors of the outcome variables beyond the variance explained by negative mood (See Table S1 - Supplementary Materials). These findings suggest that negative mood did not fully account for the associations of sleep disruption with rumination, psychotic-like experiences, and somatic symptoms.

Daily reductions in sleep duration are followed by increased rumination the next day. To examine the links between subjective reports of sleep duration and negative psychological experiences, we regressed sleep duration on average, and daily changes in sleep duration on reports of rumination, psychotic-like experiences and somatic complaints. Average sleep duration was not significantly associated with rumination. On the other hand, daily fluctuations in sleep duration (more specifically, relatively shorter durations of sleep) within-individuals were associated with increased rumination the following day. Average sleep duration or the daily changes in sleep duration within-individuals were not significantly predictive of psychotic-like experiences and somatic complaints. In sum, these findings do not indicate that individual differences in sleep duration are linked to individual differences in negative daytime 
experiences, but within the same individuals, daily reductions in sleep duration are predictive of increased rumination on the following day (See Table 3). Daily reductions in sleep duration remained significant predictors of rumination ( $b=-0.014, \mathrm{Cl}:-0.027--0.001, \mathrm{p}<0.05)$ even when controlling for the confounding effects of mood (See Table S2 in Supplementary Materials).

Associations between sleep duration and subsequent daytime functioning

\begin{tabular}{|c|c|c|c|c|c|c|}
\hline \multirow[b]{2}{*}{ Predictors } & \multicolumn{2}{|c|}{ Rumination } & \multicolumn{2}{|c|}{ Psychotic-like experiences } & \multicolumn{2}{|c|}{ Somatic complaints } \\
\hline & Estimates & $95 \% C I$ & Estimates & $95 \% C I$ & Estimates & $95 \% C I$ \\
\hline Intercept & 0.523 & $-0.016-1.062$ & 0.394 & $-0.289-1.076$ & 0.553 & $-0.008-1.115$ \\
\hline Age & 0.000 & $-0.005-0.005$ & -0.002 & $-0.008-0.005$ & -0.001 & $-0.006-0.004$ \\
\hline Gender (male) & $-0.114^{* *}$ & $-0.199--0.030$ & -0.054 & $-0.161-0.054$ & $-0.163^{* * *}$ & $-0.252--0.074$ \\
\hline PTSD-like symptoms & $0.008^{* * *}$ & $0.005-0.011$ & $0.011^{* * *}$ & $0.007-0.015$ & $0.007^{* * *}$ & $0.004-0.011$ \\
\hline Cognitive Disorganisation & $0.022^{* *}$ & $0.008-0.036$ & $0.038^{* * *}$ & $0.020-0.055$ & $0.032^{* * *}$ & $0.017-0.046$ \\
\hline Sleep duration (within-person mean) & -0.037 & $-0.094-0.021$ & -0.023 & $-0.096-0.049$ & -0.037 & $-0.096-0.023$ \\
\hline Sleep duration (within-person centered) & $-0.014^{*}$ & $-0.028--0.001$ & -0.011 & $-0.027-0.004$ & -0.011 & $-0.024-0.003$ \\
\hline \multicolumn{7}{|l|}{ Random Effects } \\
\hline$\sigma^{2}$ & \multicolumn{2}{|l|}{0.0853} & \multicolumn{2}{|l|}{0.0979} & \multicolumn{2}{|l|}{0.0574} \\
\hline$\tau_{00}$ & \multicolumn{2}{|l|}{0.0396 id } & \multicolumn{2}{|l|}{0.0675 id } & \multicolumn{2}{|l|}{0.0439 id } \\
\hline$\tau_{11}$ & & & \multicolumn{2}{|c|}{0.0007 id.sleep_duration_centered } & \multicolumn{2}{|c|}{0.0011 id.sleep_duration_centered } \\
\hline$\rho_{01}$ & & & \multicolumn{2}{|l|}{0.2451 id } & \multicolumn{2}{|l|}{$0.0341 \mathrm{id}$} \\
\hline $\mathrm{ICC}$ & \multicolumn{2}{|l|}{0.3173} & \multicolumn{2}{|l|}{0.4102} & \multicolumn{2}{|l|}{0.4390} \\
\hline $\mathrm{N}$ & \multicolumn{2}{|l|}{166 id } & \multicolumn{2}{|l|}{166 id } & \multicolumn{2}{|l|}{158 id } \\
\hline Observations & \multicolumn{2}{|l|}{1884} & \multicolumn{2}{|l|}{1884} & \multicolumn{2}{|l|}{1800} \\
\hline Marginal $\mathrm{R}^{2}$ / Conditional $\mathrm{R}^{2}$ & \multicolumn{2}{|c|}{$0.128 / 0.405$} & \multicolumn{2}{|c|}{$0.193 / 0.524$} & \multicolumn{2}{|c|}{$0.210 / 0.557$} \\
\hline
\end{tabular}

Table 3. Summary of mixed models examining associations between sleep duration and subsequent daytime functioning. Note that the model predicting rumination does not include a random slope as the model with the random slope had a singular fit (random slope and intercept were perfectly correlated). $\sigma 2$ : residual variance; $\tau 00$ : variance of random intercept; $\tau 11$ : variance of random slope; $\rho 01$ : correlation between random intercept and slope; ICC: intraclass correlation; N: number of participants. Marginal $\mathrm{R}_{2}$ : variance explained by fixed effects; Conditional R: variance explained by fixed and random effects. Pvalues were computed with Satterthwaite's approximation.

Negative daytime experiences do not predict sleep disruption or sleep duration the next night. To investigate bidirectional relationships regarding day-to-day associations between sleep and daytime 
experiences, we also examined the temporal associations between daytime experiences and sleep on the following night. Daily rates of rumination, psychotic-like experiences, somatic complaints, or negative mood did not significantly predict subjective sleep disruption and sleep duration the following night. On the other hand, older age was associated with poorer sleep quality (on average) during the two-week assessment period (See Table 4). In sum, whereas sleep disruption and sleep duration predicted daytime negative experiences the following day, no signs of such temporal association were observed in the other direction. 
Associations between daytime functioning and subsequent sleep

\begin{tabular}{|c|c|c|c|c|}
\hline \multirow[b]{2}{*}{ Predictors } & \multicolumn{2}{|c|}{ Sleep disruption } & \multicolumn{2}{|c|}{ Sleep duration } \\
\hline & Estimates & $95 \% C I$ & Estimates & $95 \% \mathrm{CI}$ \\
\hline Intercept & $0.349^{* *}$ & $0.106-0.592$ & $8.566^{* * *}$ & $7.782-9.350$ \\
\hline Age & $0.005^{*}$ & $0.001-0.010$ & $-0.028^{* * *}$ & $-0.041--0.015$ \\
\hline Gender (male) & -0.065 & $-0.139-0.009$ & $-0.442^{* * *}$ & $-0.681--0.204$ \\
\hline PTSD-like symptoms & -0.000 & $-0.003-0.003$ & 0.006 & $-0.004-0.016$ \\
\hline Cognitive Disorganisation & 0.007 & $-0.005-0.020$ & -0.026 & $-0.067-0.015$ \\
\hline Psychotic-like experiences (within-person mean) & 0.004 & $-0.006-0.015$ & 0.030 & $-0.003-0.063$ \\
\hline Psychotic-like experiences (within-person centered) & 0.000 & $-0.004-0.005$ & 0.008 & $-0.007-0.024$ \\
\hline Rumination (within-person mean) & 0.004 & $-0.015-0.024$ & -0.051 & $-0.115-0.012$ \\
\hline Rumination (within-person centered) & 0.003 & $-0.005-0.010$ & -0.007 & $-0.032-0.018$ \\
\hline Somatic complaints (within-person mean) & $0.021^{* *}$ & $0.007-0.035$ & -0.021 & $-0.067-0.025$ \\
\hline Somatic complaints (within-person centered) & -0.006 & $-0.014-0.001$ & 0.014 & $-0.010-0.038$ \\
\hline Mood (within-person mean) & -0.029 & $-0.065-0.008$ & 0.091 & $-0.027-0.209$ \\
\hline Mood (within-person centered) & -0.005 & $-0.018-0.007$ & 0.007 & $-0.035-0.048$ \\
\hline \multicolumn{5}{|l|}{ Random Effects } \\
\hline$\sigma^{2}$ & 0.0916 & & 0.9900 & \\
\hline$\tau_{00}$ & 0.0251 id & & 0.2556 id & \\
\hline$\tau_{11}$ & \multicolumn{4}{|c|}{0.0000 id.rumination_sum_centered } \\
\hline$\rho_{01}$ & $0.4755 \mathrm{id}$ & & & \\
\hline ICC & 0.2176 & & 0.2052 & \\
\hline $\mathrm{N}$ & 158 id & & 158 id & \\
\hline Observations & 1683 & & 1682 & \\
\hline Marginal $\mathrm{R}^{2} /$ Conditional $\mathrm{R}^{2}$ & $0.096 / 0.292$ & & $0.067 / 0.2$ & \\
\hline
\end{tabular}

Table 4. Summary of mixed models examining associations between daytime functioning and subsequent (next night's) sleep quality and duration. $\sigma 2$ : residual variance; $\tau 00$ : variance of random intercept; $\tau 11$ : variance of random slope; $\rho 01$ : correlation between random intercept and slope; ICC: intraclass correlation; $\mathrm{N}$ : number of participants. Marginal R2: variance explained by fixed effects; Conditional $\mathrm{R}_{2}$ : variance explained by fixed and random effects. P-values were computed with Satterthwaite's approximation. 


\section{Discussion}

The aim of our study was to prospectively investigate the associations between subjective sleep quality and psychological health in the context of home confinement due to the COVID-19 pandemic. More specifically, 166 individuals from three different countries (out of 717 respondents in a cross-sectional survey) filled in daily reports during two consecutive weeks. We focused our analyses on the associations at the between-individual level, as well as on the bidirectional temporal links between day-to-day variations of sleep quality and daytime rumination, psychotic-like experiences, and somatic complaints. Our findings indicate that disrupted sleep during the assessment period was associated with more negative psychological conditions such as increased rumination, more psychotic-like experiences, and somatic complaints resembling the most common symptoms of the coronavirus. Furthermore, day-to-day fluctuations covaried within individuals: a relatively poorer night of sleep predicted increased rumination, psychotic-like experiences, and somatic complaints the following day. This association appeared to be unidirectional, since daytime reports of such mental experiences were not associated with poor sleep quality on the following night.

A growing number of studies have reported an alarming increase in the prevalence of mental health complaints during the COVID-19 pandemic 6 6,10,11,15,16,53. The cross-sectional findings in our samples are in line with these first cross-sectional observations indicating clinically relevant signs of depression and posttraumatic symptomatology in approximately $20 \%$ of our respondents. Likewise, impaired sleep quality exhibited moderate associations with retrospectively assessed psychopathological measures 3,14,15 such as depression, posttraumatic symptoms, and cognitive disorganization. Since our aim was to include only healthy participants free from severe psychological conditions in our prospective study, we excluded individuals showing clinically relevant signs of depressive symptoms. Nevertheless, we retained and included in our models the scores of PTSD-like symptoms and cognitive disorganization as proxies of 
psychopathological conditions that were consistently linked to impaired sleep quality ${ }^{54-57}$, negative emotionality and reduced resilience ${ }^{47-50}$. This way, by controlling the effects of the levels of more general psychopathological traits, we could disentangle the associations of sleep quality with daytime reports that reflect rather specific mental experiences such as rumination, psychotic-like experiences, and somatic complaints during the confinement.

To evaluate if the perceived circumstances of the pandemic and the confinement influenced our variables of interest, we examined whether the country-specific and global number of deaths, as well as the time spent confined at home were predictive of daily measures of psychological functioning. Country-specific, daily numbers of deaths related to COVID-19 were predictive of worse mood, more psychotic-like experiences and somatic complaints during the same day, and poorer sleep quality the following night. As for the worldwide daily number of deaths, a less general pattern was observed. There was a positive link with more somatic complaints (the same day) and longer sleep duration the following night. Beyond the associations with COVID-19-related number of deaths, daily variations in rumination, psychotic-like experiences and somatic complaints were related to the time spent in confinement. On the one hand, we highlight a general improvement in psychological functioning with days elapsed from the introduction of the confinement, but a quadratic (U-shaped) relationship was also observed, indicating that negative psychological states seem to reappear with prolonged confinement. Although we abstain from drawing conclusions regarding the causal relationship of death rates and confinement days with psychological functioning, our results suggest that day-to-day variations of negative mental experiences were not independent of the context of the confinement and the pervasive information about the negative consequences of the COVID-19. Previous studies indicate that social medinteria exposure ${ }^{58}$ and 'local' COVID-19 death counts ${ }^{17}$ during the outbreak are risk factors for mental health problems. Our findings are in line with these reports and extend these observations by evidencing a link between death rates and 
psychological functioning on a day-to-day basis. The gradual amelioration of mental health as a function of time elapsed since the introduction of the confinement is also in line with a previous study showing a reduction of the negative psychological impact of the pandemic four weeks after its initial outbreak ${ }^{59}$. Although the number of our observations were relatively lower at later time points ( $>60$ days after the introduction of the confinement) these data indicate that a further prolongation of the confinement period could be associated with negative psychological outcomes ${ }^{7}$.

Our results unambiguously indicate that poor subjective sleep quality during the two-week assessment period was associated with increased rumination, psychotic-like experiences and somatic complaints about the most typical symptoms of the coronavirus. That is, individuals who exhibited poor subjective sleep quality on average were also more likely to exhibit negative psychological outcomes during the confinement. The outbreak of the pandemic and the extreme circumstances produced by the confinement seem to have had a profound negative impact on subjective sleep quality, and sleep problems consistently correlated with impaired psychological functioning $3,14,18,60$. Our findings based on the prospective assessment of sleep quality and mental health indices corroborate these previous results as well as the well-established role of sleep in emotional adaptation ${ }^{22,25,61}$. Furthermore, our data indicate that sleep disruption was associated with rumination, psychotic-like experiences and somatic complaints over and above the influence of psychopathological traits (PTSD-like symptoms and cognitive disorganization) as well as inter-and intra-individual variations in daily mood. This suggests that the association of sleep quality with more specific mental experiences during the confinement are not merely attributable to general levels of psychopathology and negative affect. At variance with subjective sleep quality, we did not observe similar links between sleep duration and psychological functioning. Whereas sleep deprivation and sleep restriction have been consistently associated with increased negative affect ${ }^{61}$, pain sensitivity ${ }^{62}$, and psychotic-like phenomena ${ }^{20,63}$, links between individual differences in sleep duration and negative health outcomes have only been reported in much larger samples and specifically 
in the case of extremely short and long sleep durations ${ }^{64,65}$. Moreover, studies suggest that home confinement provided more freedom to schedule bed-and wake-up times leading to an increase in the time spent in bed ${ }^{18,66}$. Time in bed however does not necessarily correlate with sleep duration, and what is more, it may reduce the perceived quality of sleep, especially in those individuals who are vulnerable to sleep problems ${ }^{26}$.

Our participants exhibited remarkable intra-individual variability in psychological measures and sleep quality during the two-week study period, allowing for the analyses of day-to-day associations between sleep and daytime mental experiences. Night-to-night variations in sleep quality predicted mental experiences the upcoming day, i.e., worse nights were predictive of increased rumination, psychotic-like experiences, and somatic complaints. Moreover, these associations remained significant after controlling for associations with daytime mood. Whereas average sleep duration was not associated with daytime psychological functioning, daily reductions in sleep durations (within-individuals) were associated with increased rumination the following day regardless of the influence of daytime mood. Noticeably, temporal associations between sleep and daytime psychological measures were not observed in the reverse direction. Simply put, worse days were not followed by worse nights. Such associations (i.e., daytime stress leading to sleep disruption the following night) have been reported in some studies ${ }^{67-69}$, but others found only unidirectional links between sleep quality and next day's psychological functioning ${ }^{33,70,71}$. Although a bidirectional relationship between sleep and daytime functioning seems plausible ${ }^{61}$, future studies and meta-analyses should corroborate if associations are present in both directions.

The global atmosphere of anxiety and the unusual circumstances of home confinement after the outbreak of the COVID-19 imposed an overwhelming mental health burden on the population. Intrusive and recurrent thoughts, cognitive disorganization and unusual experiences, and dysfunctional anxiety about contracting the coronavirus were common experiences all over the globe. Our findings indicate that 
restorative sleep could be an important factor to counteract the depletion of cognitive resources required for efficient emotional coping strategies during the challenging days of the confinement. Although our results call out for the development of sleep quality-promoting strategies to help individuals to cope better with stressful situations, their impact remains to be investigated.

\section{Methods}

Participants. Individuals ( $\mathrm{N}=728,556$ (76\%) females, age $=18-69$ years, Mean $_{\text {age }}=28.5, \mathrm{SD}_{\text {age }}=10.09$ ) willing to participate in our study were selected from three European countries in which restrictive confinement measures were adopted. The majority of the respondents were young, university students and their family members. Participants were contacted by advertisements placed on social media, and through the web pages and mailing services of the participating universities (Université Libre de Bruxelles, Belgium, Eötvös Loránd University, Hungary, and Autonomous University of Madrid, Spain). In the first phase of the study, participants were asked to complete an online questionnaire including self-report scales of standardized questionnaires measuring sleep quality ${ }^{72}$, depression ${ }^{73}$, schizotypy ${ }^{74}$, and symptoms of PTSD ${ }^{52}$. Demographic variables and items related to events (e.g. tested positive for COVID19) and experiences related to the confinement (e.g. household, school- and work-related stress) were also assessed. This cross-sectional questionnaire served us to screen and select the participants for the second, prospective phase of the study. Individuals with current or prior history of neurological, psychiatric, or chronic somatic diseases, scoring above 12 on the short form of the Beck Depression Inventory $\left(\mathrm{BDI}-13^{73}\right)$, or taking medication (except contraceptives) were excluded. In addition, we excluded participants who reported to be previously diagnosed with COVID-19 or believed that they currently suffered or had suffered but recovered from the virus. Individuals fulfilling the inclusion criteria and willing to continue participation ( $N=246$ ) were selected for the prospective study phase. 184 individuals (146 (79 \%) females, age 18-69 years, $M_{\text {age }}=26.28, S D=7.42$ ) were assigned for the second phase of the study assessing daily questionnaires during two weeks. Informed consents were obtained, and the study was approved by the corresponding local ethical committees of the three participating universities, the local Ethics Committee of the Université Libre de Bruxelles, the Ethics Committee of the Eötvös Loránd University, and the Ethics Committee of the Autonomous University of Madrid, respectively.

Procedure. The study consisted of three phases: 1) the administration of the cross-sectional questionnaire to screen and enroll potential participants, 2) the prospective study phase asking participants to complete short questionnaires twice a day during two consecutive weeks, and 3) the debriefing phase. The same questionnaire-batteries were used for the Belgian, Hungarian, and Spanish samples using the standardized or available versions in English, Hungarian, and Spanish, respectively. Items that were not available in Spanish or Hungarian were translated by the authors. Research assistants invited the selected participants to sign up for the prospective phase of the study through a dedicated website where only the invited participants could register and approve their registration via a two-step authentication process. 
Participants signed up between the $9^{\text {th }}$ of April 2020 and the 14t of May 2020 for the two-week assessment period (See Figure 4 showing the two-week assessment period within the timeline of the pandemic). Daily questionnaires requiring approximately 3-5 minutes per day were sent to participants via the online interface. A morning questionnaire was available between 5:00 and 12:30 am, and an evening questionnaire was accessible between $18: 00 \mathrm{pm}$ and 03:00 am. Participants were instructed to complete the morning questionnaire upon awakening, and to complete the second questionnaire always before going to sleep. A reminder email of the morning questionnaire was sent between 7:00-9:00 am, and a reminder of the second questionnaire was sent between 19:00-20:00 pm. The two-week long prospective study period started with the first morning questionnaire the day after the participant completed the registration and ended on the $14^{\text {th }}$ day with the last evening questionnaire. Morning questionnaires assessed subjective sleep quality and sleep schedules (bedtime, sleep latency, and wake up time). The evening questionnaires consisted of items measuring mood, rumination, psychotic-like experiences and somatic complaints comprising the most typical symptoms of the COVID-19. Participants were also asked to report daily caffeine intake and alcohol consumption and their overall satisfaction with daily activities (work, physical activity, social interactions, media use, etc.). After finishing the last questionnaire, participants were instructed to access the debriefing that consisted of a final phase providing the possibility for the participants to ask questions regarding the study and to report any issue that they considered important. Moreover, they were asked to report if they had been tested positive for COVID19 and if they assumed that they had contracted the virus during the study period.

Cross-sectional measures. The PTSD Checklist from the DSM-5 (PCL-5) was used to assess PTSD-like symptoms ${ }^{52}$. This 20 -item self-report questionnaire that assesses DSM-5 symptoms of PTSD is suitable to screen individuals for PTSD, but it can also be applied from a dimensional point of view to quantify the severity of symptoms of stress in clinical and subclinical populations ${ }^{75}$. Symptoms of depression were assessed with the short form of the Beck Depression Inventory (BDI-13). The questionnaire is suitable to screen individuals with mild, moderate, or severe symptoms of depression ${ }^{73}$. Sleep disturbances were measured by the respective subscale of the Pittsburgh Sleep Quality Index (PSQI ${ }^{72}$ ), a questionnaire used widely to evaluate subjective sleep quality. The sleep disturbances subscale focuses on subjective sleep complaints, such as having difficulties falling asleep, nocturnal awakenings, pain and discomfort during bedtime, as well as daytime sleepiness and fatigue. We used the Cognitive Disorganization scale of the Oxford-Liverpool Inventory of Feelings and Experiences (O-LIFE) to evaluate the cognitive aspects of traitlike schizotypy ${ }^{74}$. The 11 yes/no items of the scale assess difficulties in attention, concentration, decision making, and social functioning. The levels of school- and/or work-related stress experienced as a consequence of the confinement were assessed by seven-point Likert-scales (e.g. 0 - I experience much less stress since the introduction of the confinement, $6-$ I experience much more stress...).

Morning questionnaire. Participants completed the Groningen Sleep Quality Scale (GSQS) ${ }^{76}$ each morning. The questionnaire consists of 14 binary items that measure the extent of subjective sleep fragmentation. Previous studies indicate that the questionnaire is an adequate tool for assessing subjective sleep disruption ${ }^{77}$. Additionally, participants were asked to report their bedtime, sleep latency (the time they needed to fall asleep) and wake up time. These responses were used to compute subjective sleep duration (i.e. the time elapsed between sleep onset and morning awakening).

Evening questionnaire. To measure ruminative, perseverative and intrusive thoughts, items assessing state-rumination (developed by Kocsel and colleagues ${ }^{38}$ ) were adapted to the context of the confinement. Three statements ("I was not able to get certain thoughts out of my mind."; "I kept thinking about something over and over again."; "I had difficulties in suppressing my thoughts about the current situation.") were rated on a 4-point Likert scale. Psychotic-like experiences were assessed with a scale 
consisting of eight items that were originally adapted from two validated instruments ${ }^{40,78}$. The items (rated on an 8-point Likert scale) covered perceptual anomalies (e.g. "Familiar things have seemed strange or unusual"), cognitive disorganization (e.g. "I have found it difficult to think clearly") and paranoid ideation (e.g. "I think people have been saying or doing things to annoy me."). The scale showed good psychometric properties and proved to be effective for the daily assessment of psychotic-like experiences in a previous study ${ }^{41}$. Somatic complaints were measured by items adopted from the Patient Health Questionnaire (PHQ-15) ${ }^{44}$. The PHQ-15 was designed to measure the prevalence of the most common body symptoms (e.g., headache, nausea, etc.). Here, we used items that overlapped with the most typical symptoms of the coronavirus such as stomach pain, headache, chest pain, dizziness, low energy, muscle pain, shortness of breath, and we added an extra item concerning the experience of dry cough or sore throat. Daytime mood was assessed by a single item ("Throughout the day my mood was...") rated on an 8-point Likert scale (0 - Extremely negative: sad, negative, distressed; 7 - Extremely positive: happy, joyful, relaxed).

Statistical analyses. In order to account for the nested structure of the data (repeated measures within participants), linear mixed models were fitted using the Ime4 package ${ }^{79}$ in R (v3.6.3). Every model included a random intercept and random slope per participant for the within-person centered time-varying predictor. In case of convergence issues, random slopes were removed, and a random intercept-only model was fitted. We disentangled between- and within-person effects of time-varying variables obtained from the prospective study ${ }^{80}$. Within-person averages over the two-week study period were entered to assess between-person effects (i.e. to capture differences between participants scoring high or low on a measure on average), while within-person centering was applied to examine within-person effects (i.e. to capture correlates of day-to-day deviation from the participant's average on a measure). When we fitted models that included somatic complaints, we excluded data from eight participants who indicated at debriefing phase that they believed they had contracted the coronavirus during their participation in the study.

First, we investigated how events associated with the pandemic predicted daytime functioning, sleep disruption, and sleep duration. Predictors were country-wise and worldwide numbers of COVID-related deaths for the given day (accessed from https://www.worldometers.info/coronavirus/coronavirus-deathtoll/ as of 19th May 2020). In order to facilitate interpretation of coefficient estimates, the number of country-wise and global deaths per day were scaled with a division by 10 and 1000, respectively. Additionally, we calculated the number of days since the introduction of confinement in the country of the participant (Belgium: 14th March 2020, Hungary: 28th March 2020, Spain: 17th March 2020). Time spent in confinement was expressed in weeks (number of days / 7) in order to have this variable on a scale comparable to the other predictors (this was done to facilitate model fitting and interpretation of coefficients). In order to capture non-linear effects, time spent in confinement was entered as a linear and as a quadratic term as well. Age, gender, PTSD-like symptoms (PCL-5 sum score) and cognitive disorganization were entered as person-specific time-invariant predictors. Dependent variables were the sum scores derived from the prospective measurements of rumination, psychotic-like experiences and somatic complaints during the day, and sleep disruption and sleep duration the following night. 


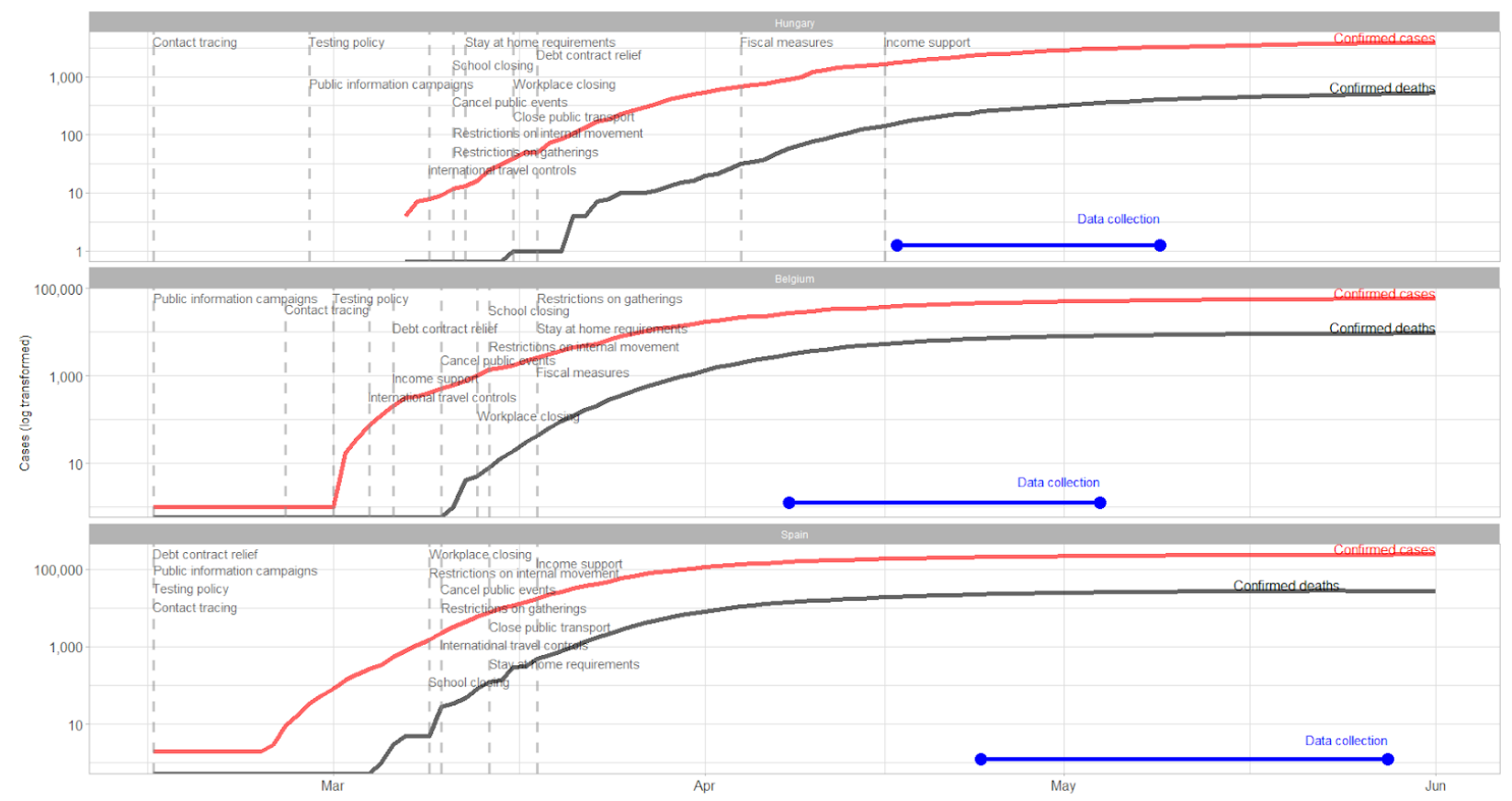

Figure 4. The plot shows the progress - confirmed cases and deaths - of the COVID-19 pandemic in three European countries. Dashed lines show the introduction of specific countermeasures. The blue line segment shows when the data collection took place. Data from: Hale, Thomas, Sam Webster, Anna Petherick, Toby Phillips, and Beatriz Kira (2020). Oxford COVID-19 Government Response Tracker, Blavatnik School of Government. https://covidtracker.bsg.ox.ac.uk/

Then, we analyzed the relationship between sleep disruption / duration and functioning on the next day. Dependent variables were the sum scores derived from the prospective measurements of rumination, psychotic-like experiences, and somatic complaints. As diagnostic plots suggested that model residuals were not normally distributed, each dependent variable was log10-transformed, after which model residuals did not appear to strongly violate normality. Age, gender, PTSD-like symptoms (PCL-5 sum score), cognitive disorganization and within-person mean of subjective sleep disruption (GSQS sum score) / duration were entered as level-2 predictors, and within-person centered subjective sleep quality / duration were entered as a level-1 predictor in the models.

In the analysis of the relationship between daytime functioning and sleep disruption / duration the following night, the dependent variables were sleep quality (GSQS sum score) and sleep duration. Age, gender, PTSD-like symptoms (PCL-5 sum score), cognitive disorganization and within-person means of mood, rumination, psychotic-like experiences and somatic complaints were entered as level-2 predictors, and within-person centered mood, rumination, psychotic-like experiences and somatic complaints (of the day before sleep) were entered as level-1 predictors.

\section{Open data and analysis scripts}

Anonymized data and analysis scripts can be found on the project's OSF page:

https://osf.io/xabwe/?view only=f319528ac4c54e08bb041c788dcb6339 


\section{Acknowledgments}

The authors thank Róbert Báthori who designed the user interface and layout and created the online platform for data collection.

\section{References}

1. Zhou, F. et al. Clinical course and risk factors for mortality of adult inpatients with COVID-19 in Wuhan, China: a retrospective cohort study. The Lancet 395, 1054-1062 (2020).

2. Organization, W. H. Coronavirus disease 2019 (COVID-19): situation report, 72. (2020).

3. Rajkumar, R. P. COVID-19 and mental health: A review of the existing literature. Asian J Psychiatr 52, 102066 (2020).

4. Dutheil, F., Mondillon, L. \& Navel, V. PTSD as the second tsunami of the SARS-Cov-2 pandemic. Psychological Medicine 1-2 (undefined/ed) doi:10.1017/S0033291720001336.

5. Bavel, J. J. V. et al. Using social and behavioural science to support COVID-19 pandemic response. Nature Human Behaviour 4, 460-471 (2020).

6. Huang, Y. \& Zhao, N. Chinese mental health burden during the COVID-19 pandemic. Asian J Psychiatr 51, 102052 (2020).

7. Brooks, S. K. et al. The psychological impact of quarantine and how to reduce it: rapid review of the evidence. The Lancet (2020).

8. Liu, N. et al. Prevalence and predictors of PTSS during COVID-19 outbreak in China hardest-hit areas: Gender differences matter. Psychiatry research 112921 (2020).

9. Lai, J. et al. Factors Associated With Mental Health Outcomes Among Health Care Workers Exposed to Coronavirus Disease 2019. JAMA Netw Open 3, e203976-e203976 (2020).

10. Kaparounaki, C. K. et al. University students' mental health amidst the COVID-19 quarantine in Greece. Psychiatry Research 113111 (2020). 
11. Odriozola-González, P., Planchuelo-Gómez, Á., Irurtia, M. J. \& de Luis-García, R. Psychological effects of the COVID-19 outbreak and lockdown among students and workers of a Spanish university. Psychiatry Research 113108 (2020).

12. Zhao, X., Lan, M., Li, H. \& Yang, J. Perceived Stress and Sleep Quality Among the Non-diseased General Public in China During the 2019 Coronavirus Disease: A Moderated Mediation Model. Sleep Medicine (2020).

13. Wang, C. et al. Immediate psychological responses and associated factors during the initial stage of the 2019 coronavirus disease (COVID-19) epidemic among the general population in China. International journal of environmental research and public health 17, 1729 (2020).

14. Casagrande, M., Favieri, F., Tambelli, R. \& Forte, G. The enemy who sealed the world: Effects quarantine due to the COVID-19 on sleep quality, anxiety, and psychological distress in the Italian population. Sleep Medicine (2020).

15. Huang, Y. \& Zhao, N. Generalized anxiety disorder, depressive symptoms and sleep quality during COVID-19 outbreak in China: a web-based cross-sectional survey. Psychiatry Research 288, 112954 (2020).

16. Lin, L. et al. The immediate impact of the 2019 novel coronavirus (COVID-19) outbreak on subjective sleep status. Sleep Medicine (2020).

17. Zhang, Y., Zhang, H., Ma, X. \& Di, Q. Mental Health Problems during the COVID-19 Pandemics and the Mitigation Effects of Exercise: A Longitudinal Study of College Students in China. International Journal of Environmental Research and Public Health 17, 3722 (2020).

18. Cellini, N., Canale, N., Mioni, G. \& Costa, S. Changes in sleep pattern, sense of time and digital media use during COVID-19 lockdown in Italy. Journal of Sleep Research e13074 (2020). 
19. van Liempt, S., van Zuiden, M., Westenberg, H., Super, A. \& Vermetten, E. Impact of impaired sleep on the development of PTSD symptoms in combat veterans: a prospective longitudinal cohort study. Depress Anxiety 30, 469-474 (2013).

20. Barton, J., Kyle, S. D., Varese, F., Jones, S. H. \& Haddock, G. Are sleep disturbances causally linked to the presence and severity of psychotic-like, dissociative and hypomanic experiences in nonclinical populations? A systematic review. Neurosci Biobehav Rev 89, 119-131 (2018).

21. Baglioni, C. et al. Insomnia as a predictor of depression: a meta-analytic evaluation of longitudinal epidemiological studies. Journal of affective disorders 135, 10-19 (2011).

22. Walker, M. P. \& van der Helm, E. Overnight therapy? The role of sleep in emotional brain processing. Psychol Bull 135, 731-748 (2009).

23. Simon, E. B., Rossi, A., Harvey, A. G. \& Walker, M. P. Overanxious and underslept. Nature Human Behaviour 1-11 (2019) doi:10.1038/s41562-019-0754-8.

24. Wassing, R. et al. Restless REM Sleep Impedes Overnight Amygdala Adaptation. Curr. Biol. 29, 2351-2358.e4 (2019).

25. Deliens, G., Gilson, M. \& Peigneux, P. Sleep and the processing of emotions. Exp Brain Res 232, 1403-1414 (2014).

26. Altena, E. et al. Dealing with sleep problems during home confinement due to the COVID-19 outbreak: Practical recommendations from a task force of the European CBT-I Academy. Journal of Sleep Research e13052 (2020).

27. Babkoff, H., Weller, A. \& Lavidor, M. A comparison of prospective and retrospective assessments of sleep. Journal of Clinical Epidemiology 49, 455-460 (1996).

28. Sato, H. \& Kawahara, J. Selective bias in retrospective self-reports of negative mood states. Anxiety, Stress, \& Coping 24, 359-367 (2011). 
29. Schroeder, D. H. \& Costa, P. T. Influence of life event stress on physical illness: Substantive effects or methodological flaws? Journal of Personality and Social Psychology 46, 853-863 (1984).

30. Solhan, M. B., Trull, T. J., Jahng, S. \& Wood, P. K. Clinical assessment of affective instability: Comparing EMA indices, questionnaire reports, and retrospective recall. Psychological Assessment 21, 425-436 (2009).

31. Kasanova, Z., Hajdúk, M., Thewissen, V. \& Myin-Germeys, I. Temporal associations between sleep quality and paranoia across the paranoia continuum: An experience sampling study. Journal of Abnormal Psychology 129, 122-130 (2020).

32. Hennig, T. \& Lincoln, T. M. Sleeping Paranoia Away? An Actigraphy and Experience-Sampling Study with Adolescents. Child Psychiatry Hum Dev 49, 63-72 (2018).

33. Simor, P., Krietsch, K. N., Köteles, F. \& McCrae, C. S. Day-to-day variation of subjective sleep quality and emotional states among healthy university students-a 1-week prospective study. International journal of behavioral medicine 22, 625-634 (2015).

34. Kramer, I. et al. Time-Lagged Moment-to-Moment Interplay Between Negative Affect and Paranoia: New Insights in the Affective Pathway to Psychosis. Schizophr Bull 40, 278-286 (2014).

35. Fleeson, W. Situation-Based Contingencies Underlying Trait-Content Manifestation in Behavior. Journal of Personality 75, 825-862 (2007).

36. Nolen-Hoeksema, S., Wisco, B. E. \& Lyubomirsky, S. Rethinking Rumination: Perspectives on Psychological Science (2008).

37. Nolen-Hoeksema, S. \& Watkins, E. R. A Heuristic for Developing Transdiagnostic Models of Psychopathology: Explaining Multifinality and Divergent Trajectories. Perspectives on Psychological Science (2011) doi:10.1177/1745691611419672.

38. Kocsel, N. et al. The association between perseverative cognition and resting heart rate variability: A focus on state ruminative thoughts. Biological Psychology 145, 124-133 (2019). 
39. van Os, J., Linscott, R. J., Myin-Germeys, I., Delespaul, P. \& Krabbendam, L. A systematic review and meta-analysis of the psychosis continuum: evidence for a psychosis proneness-persistenceimpairment model of psychotic disorder. Psychol Med 39, 179-195 (2009).

40. Cristóbal-Narváez, P. et al. The role of stress-regulation genes in moderating the association of stress and daily-life psychotic experiences. Acta Psychiatr Scand 136, 389-399 (2017).

41. Simor, P., Báthori, N., Nagy, T. \& Polner, B. Poor sleep quality predicts psychotic-like symptoms: an experience sampling study in young adults with schizotypal traits. Acta Psychiatrica Scandinavica 140, 135-146 (2019).

42. Collip, D. et al. Daily cortisol, stress reactivity and psychotic experiences in individuals at above average genetic risk for psychosis. Psychological Medicine 41, 2305-2315 (2011).

43. Lee, S. A., Jobe, M. C. \& Mathis, A. A. Mental health characteristics associated with dysfunctional coronavirus anxiety. Psychol Med 1-2 (2020) doi:10.1017/S003329172000121X.

44. Kroenke, K., Spitzer, R. L. \& Williams, J. B. The PHQ-15: validity of a new measure for evaluating the severity of somatic symptoms. Psychosomatic medicine 64, 258-266 (2002).

45. Finan, P. H., Goodin, B. R. \& Smith, M. T. The Association of Sleep and Pain: An Update and a Path Forward. The Journal of Pain 14, 1539-1552 (2013).

46. Buchanan Diana Taibi et al. Sleep Measures Predict Next-Day Symptoms in Women with Irritable Bowel Syndrome. Journal of Clinical Sleep Medicine 10, 1003-1009.

47. DiCorcia, J. A. \& Tronick, E. Quotidian resilience: Exploring mechanisms that drive resilience from a perspective of everyday stress and coping. Neuroscience \& Biobehavioral Reviews 35, 1593-1602 (2011).

48. CICCHETTI, D. Resilience under conditions of extreme stress: a multilevel perspective. World Psychiatry 9, 145-154 (2010). 
49. Polner, B. et al. The network structure of schizotypy in the general population. Eur Arch Psychiatry Clin Neurosci (2019) doi:10.1007/s00406-019-01078-x.

50. Grant, P. \& Hennig, J. Schizotypy, social stress and the emergence of psychotic-like states - A case for benign schizotypy? Schizophrenia Research 216, 435-442 (2020).

51. Furlanetto, L. M., Mendlowicz, M. V. \& Bueno, J. R. The validity of the Beck Depression Inventory-Short Form as a screening and diagnostic instrument for moderate and severe depression in medical inpatients. Journal of affective disorders 86, 87-91 (2005).

52. Blevins, C. A., Weathers, F. W., Davis, M. T., Witte, T. K. \& Domino, J. L. The posttraumatic stress disorder checklist for DSM-5 (PCL-5): Development and initial psychometric evaluation. Journal of Traumatic Stress 28, 489-498 (2015).

53. Kwong, A. S. F. et al. Mental health during the COVID-19 pandemic in two longitudinal UK population cohorts. medRxiv 2020.06.16.20133116 (2020) doi:10.1101/2020.06.16.20133116.

54. Tempesta, D., Curcio, G., De Gennaro, L. \& Ferrara, M. Long-term impact of earthquakes on sleep quality. PLOS ONE 8, e55936 (2013).

55. Charuvastra, A. \& Cloitre, M. Safe enough to sleep: sleep disruptions associated with trauma, posttraumatic stress, and anxiety in children and adolescents. Child and Adolescent Psychiatric Clinics of North America 18, 877-891 (2009).

56. Ross, R. J., Ball, W. A., Sullivan, K. A. \& Caroff, S. N. Sleep disturbance as the hallmark of posttraumatic stress disorder. Am J Psychiatry 146, 697-707 (1989).

57. Reeve, S., Sheaves, B. \& Freeman, D. The role of sleep dysfunction in the occurrence of delusions and hallucinations: A systematic review. Clin Psychol Rev 42, 96-115 (2015).

58. Gao, J. et al. Mental health problems and social media exposure during COVID-19 outbreak. Plos one 15, e0231924 (2020). 
59. Wang, C. et al. A longitudinal study on the mental health of general population during the COVID-19 epidemic in China. Brain, behavior, and immunity (2020).

60. Li, D.-J. et al. Covid-19-related factors associated with sleep disturbance and suicidal thoughts among the Taiwanese public: a facebook survey. International journal of environmental research and public health 17, 4479 (2020).

61. Simon, E. B., Vallat, R., Barnes, C. M. \& Walker, M. P. Sleep Loss and the Socio-Emotional Brain. Trends in Cognitive Sciences (2020).

62. Schrimpf, M. et al. The effect of sleep deprivation on pain perception in healthy subjects: a meta-analysis. Sleep Medicine 16, 1313-1320 (2015).

63. Reeve, S., Emsley, R., Sheaves, B. \& Freeman, D. Disrupting Sleep: The Effects of Sleep Loss on Psychotic Experiences Tested in an Experimental Study With Mediation Analysis. Schizophr Bull 44, $662-671$ (2018).

64. Cappuccio, F. P., D’Elia, L., Strazzullo, P. \& Miller, M. A. Sleep Duration and All-Cause Mortality: A Systematic Review and Meta-Analysis of Prospective Studies. Sleep 33, 585-592 (2010).

65. Itani, O., Jike, M., Watanabe, N. \& Kaneita, Y. Short sleep duration and health outcomes: a systematic review, meta-analysis, and meta-regression. Sleep Medicine 32, 246-256 (2017).

66. Marelli, S. et al. Impact of COVID-19 lockdown on sleep quality in university students and administration staff. Journal of Neurology 1-8 (2020).

67. \AAkerstedt, T. et al. Predicting sleep quality from stress and prior sleep-a study of day-to-day covariation across six weeks. Sleep medicine 13, 674-679 (2012).

68. Pillai, V., Steenburg, L. A., Ciesla, J. A., Roth, T. \& Drake, C. L. A seven day actigraphy-based study of rumination and sleep disturbance among young adults with depressive symptoms. Journal of Psychosomatic Research 77, 70-75 (2014). 
69. Doane, L. D. \& Thurston, E. C. Associations among sleep, daily experiences, and loneliness in adolescence: Evidence of moderating and bidirectional pathways. Journal of Adolescence 37, 145-154 (2014).

70. Bouwmans, M. E. J., Bos, E. H., Hoenders, H. J. R., Oldehinkel, A. J. \& de Jonge, P. Sleep quality predicts positive and negative affect but not vice versa. An electronic diary study in depressed and healthy individuals. Journal of Affective Disorders 207, 260-267 (2017).

71. Galambos, N. L., Vargas Lascano, D. I., Howard, A. L. \& Maggs, J. L. Who sleeps best? Longitudinal patterns and covariates of change in sleep quantity, quality, and timing across four university years. Behavioral Sleep Medicine 11, 8-22 (2013).

72. Buysse, D. J., Reynolds, C. F., Monk, T. H., Berman, S. R. \& Kupfer, D. J. The Pittsburgh Sleep Quality Index: a new instrument for psychiatric practice and research. Psychiatry Res 28, 193-213 (1989).

73. Beck, A. T. \& Beck, R. W. Screening Depressed Patients in Family Practice. Postgraduate Medicine 52, 81-85 (1972).

74. Mason, O., Linney, Y. \& Claridge, G. Short scales for measuring schizotypy. Schizophrenia research 78, 293-296 (2005).

75. McCubbin, J. A. et al. Subclinical Posttraumatic Stress Disorder Symptoms: Relationships with Blood Pressure, Hostility, and Sleep. Cardiovasc Psychiatry Neurol 2016, (2016).

76. Meijman, T. F., de Vries-Griever, A. H., De Vries, G. \& Kampman, R. The evaluation of the Groningen sleep quality scale. Groningen: Heymans Bulletin (HB 88-13-EX) 2006, (1988).

77. Simor, P., Köteles, F., Bódizs, R. \& Bárdos, G. A questionnaire based study of subjective sleep quality: the psychometric evaluation of the Hungarian version of the Groningen Sleep Quality Scale. Mentálhigiéné és Pszichoszomatika 10, 249-261 (2009). 
78. Mason, O. J., Morgan, C. J. M., Stefanovic, A. \& Curran, H. V. The psychotomimetic states inventory (PSI): measuring psychotic-type experiences from ketamine and cannabis. Schizophr. Res. $103,138-142$ (2008).

79. Bates, D., Mächler, M., Bolker, B. \& Walker, S. Fitting Linear Mixed-Effects Models Using Ime4. Journal of Statistical Software 67, 1-48 (2015).

80. Wang, L. P. \& Maxwell, S. E. On disaggregating between-person and within-person effects with longitudinal data using multilevel models. Psychological methods 20, 63 (2015). 


\section{Supplementary Materials}
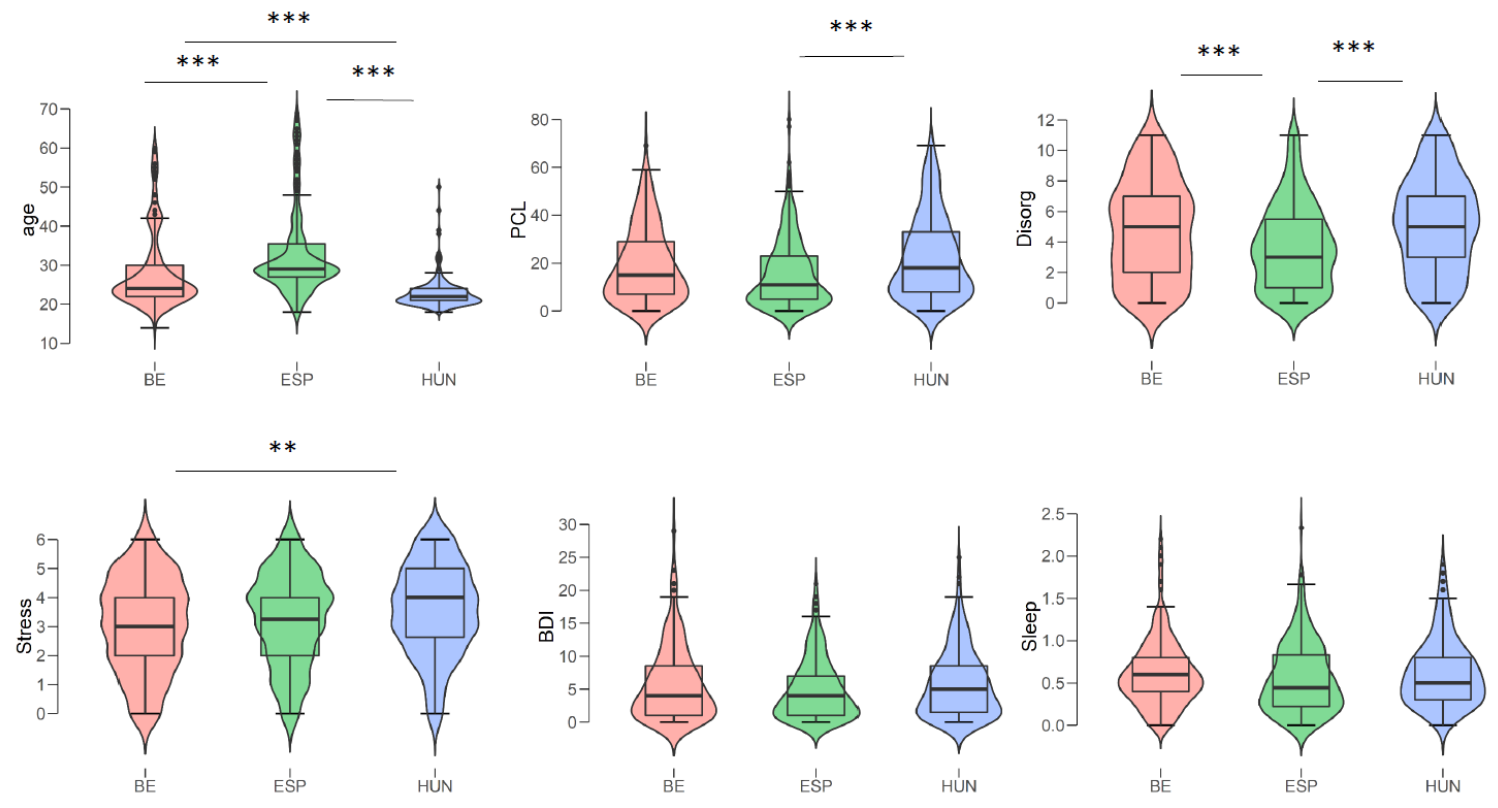

S1. Cross-sectional comparisons across the three sites, Belgium $(\mathbf{N}=\mathbf{2 2 7})$, Spain $(\mathbf{N}=\mathbf{2 8 3})$ and Hungary $(\mathbf{N}=\mathbf{2 0 7})$. Age showed the most pronounced difference across the three countries (BE vs ESP: $t=-5.41, d=-0.41, p<0.0001$; $B E$ vs HUN $t=6.14, d=0.71, p<0.0001$; ESP vs HUN $t=11.73, d=1.11, p<0.0001)$. Posttraumatic symptoms $(P C L)$, Cognitive Disorganization (Disorg), and the increase in perceived stress due to the COVID-19 (Stress) exhibited significant differences across countries. Depression (BDI), and Sleep disturbance (Sleep) did not significantly differ across countries. The effect size (Cohen's d) of the differences marked as significant ranged between 0.3 and 0.47$)$. ${ }^{* * *}$ - FDR corrected $p<0.001,{ }^{* *}-$ FDR corrected $p<$ 0.01 . 
Associations between sleep quality and subsequent daytime functioning controlled for mood

\begin{tabular}{|c|c|c|c|c|c|c|}
\hline \multirow[b]{2}{*}{ Predictors } & \multicolumn{2}{|c|}{ Rumination } & \multicolumn{2}{|c|}{ Psychotic-like experiences } & \multicolumn{2}{|c|}{ Somatic complaints } \\
\hline & Estimates & $95 \% C I$ & Estimates & $95 \% C I$ & Estimates & $95 \% C I$ \\
\hline Intercept & $0.430^{* *}$ & $0.166-0.693$ & $0.380^{*}$ & $0.055-0.705$ & $0.417^{* *}$ & $0.161-0.673$ \\
\hline Age & 0.000 & $-0.004-0.005$ & -0.003 & $-0.009-0.002$ & -0.003 & $-0.007-0.002$ \\
\hline Gender (male) & $-0.093^{*}$ & $-0.174--0.012$ & -0.017 & $-0.117-0.083$ & $-0.110^{* *}$ & $-0.190--0.030$ \\
\hline PTSD-like symptoms & $0.007^{* * *}$ & $0.004-0.010$ & $0.009^{* * *}$ & $0.005-0.013$ & $0.006^{* * *}$ & $0.003-0.009$ \\
\hline Cognitive Disorganisation & $0.014^{*}$ & $0.000-0.029$ & $0.025^{* *}$ & $0.007-0.042$ & $0.021^{* *}$ & $0.007-0.035$ \\
\hline Sleep quality (within-person mean) & $0.022^{*}$ & $0.001-0.042$ & $0.051^{* * *}$ & $0.026-0.076$ & $0.051^{* * *}$ & $0.031-0.071$ \\
\hline Sleep quality (within-person centered) & $0.007^{* *}$ & $0.002-0.013$ & $0.005^{*}$ & $0.000-0.010$ & $0.005^{*}$ & $0.000-0.010$ \\
\hline Positive mood (within-person mean) & $-0.049^{*}$ & $-0.090--0.009$ & -0.045 & $-0.095-0.005$ & $-0.046^{*}$ & $-0.085--0.007$ \\
\hline Positive mood (within-person centered) & $-0.061^{* * *}$ & $-0.071--0.051$ & $-0.088^{* * *}$ & $-0.098--0.077$ & $-0.050^{* * *}$ & $-0.058--0.041$ \\
\hline \multicolumn{7}{|l|}{ Random Effects } \\
\hline$\sigma^{2}$ & 0.0775 & & 0.0852 & & 0.0528 & \\
\hline$\tau_{00}$ & $0.0374 \mathrm{id}$ & & 0.0596 id & & 0.0355 id & \\
\hline$\tau_{11}$ & \multicolumn{4}{|c|}{0.0001 id.gsqs_sum_centered } & \multicolumn{2}{|c|}{0.0002 id.gsqs_sum_centered } \\
\hline$\rho_{01}$ & \multicolumn{2}{|l|}{$0.2212 \mathrm{id}$} & & & \multicolumn{2}{|l|}{0.1885 id } \\
\hline ICC & \multicolumn{2}{|l|}{0.3312} & \multicolumn{2}{|l|}{0.4116} & \multicolumn{2}{|l|}{0.4104} \\
\hline $\mathrm{N}$ & \multicolumn{2}{|l|}{166 id } & \multicolumn{2}{|l|}{166 id } & \multicolumn{2}{|l|}{158 id } \\
\hline Observations & \multicolumn{2}{|l|}{1884} & \multicolumn{2}{|l|}{1884} & \multicolumn{2}{|l|}{1800} \\
\hline Marginal $\mathrm{R}^{2}$ / Conditional $\mathrm{R}^{2}$ & \multicolumn{2}{|c|}{$0.193 / 0.460$} & \multicolumn{2}{|c|}{$0.293 / 0.584$} & \multicolumn{2}{|c|}{$0.309 / 0.593$} \\
\hline
\end{tabular}

Table S1. Summary of mixed models examining associations between sleep quality and subsequent daytime functioning, controlled for mood. $\sigma 2$ : residual variance; $\tau 00$ : variance of random intercept; $\tau 11$ : variance of random slope; $\rho 01$ : correlation between random intercept and slope; ICC: intraclass correlation; N: number of participants. Marginal R2: variance explained by fixed effects; Conditional $\mathrm{R}_{2}$ : variance explained by fixed and random effects. P-values were computed with Satterthwaite's approximation. 
Associations between sleep duration and subsequent daytime functioning controlled for mood

\begin{tabular}{|c|c|c|c|c|c|c|}
\hline \multirow[b]{2}{*}{ Predictors } & \multicolumn{2}{|c|}{ Rumination } & \multicolumn{2}{|c|}{ Psychotic-like experiences } & \multicolumn{2}{|c|}{ Somatic complaints } \\
\hline & Estimates & $95 \% \mathrm{CI}$ & Estimates & $95 \% \mathrm{CI}$ & Estimates & $95 \% \mathrm{CI}$ \\
\hline Intercept & $0.760^{* *}$ & $0.209-1.311$ & 0.667 & $-0.034-1.369$ & $0.811^{* *}$ & $0.244-1.379$ \\
\hline Age & 0.000 & $-0.005-0.005$ & -0.002 & $-0.008-0.004$ & -0.001 & $-0.006-0.004$ \\
\hline Gender (male) & $-0.122^{* *}$ & $-0.205--0.039$ & -0.063 & $-0.169-0.043$ & $-0.171^{* * *}$ & $-0.258--0.084$ \\
\hline PTSD-like symptoms & $0.008^{* * *}$ & $0.005-0.011$ & $0.011^{* * *}$ & $0.007-0.015$ & $0.007^{* * *}$ & $0.004-0.011$ \\
\hline Cognitive Disorganisation & $0.016^{*}$ & $0.002-0.030$ & $0.030^{* *}$ & $0.012-0.049$ & $0.024^{* *}$ & $0.010-0.039$ \\
\hline Sleep duration (within-person mean) & -0.029 & $-0.085-0.028$ & -0.016 & $-0.088-0.056$ & -0.027 & $-0.085-0.031$ \\
\hline Sleep duration (within-person centered) & $-0.014^{*}$ & $-0.027--0.001$ & -0.012 & $-0.026-0.003$ & -0.011 & $-0.024-0.001$ \\
\hline Positive mood (within-person mean) & $-0.058^{* *}$ & $-0.098--0.018$ & $-0.064^{*}$ & $-0.115--0.012$ & $-0.065^{* *}$ & $-0.106--0.023$ \\
\hline Positive mood (within-person centered) & $-0.062^{* * *}$ & $-0.072--0.052$ & $-0.089^{* * *}$ & $-0.099--0.078$ & $-0.052^{* * *}$ & $-0.060--0.043$ \\
\hline \multicolumn{7}{|l|}{ Random Effects } \\
\hline$\sigma^{2}$ & 0.0787 & & 0.0848 & & 0.0529 & \\
\hline$\tau_{00}$ & $0.0382 \mathrm{id}$ & & $0.0662 \mathrm{id}$ & & 0.0418 id & \\
\hline$\tau_{11}$ & & & 0.0004 id.sle & p_duration_centered & 0.0010 id.sle & P_duration_centered \\
\hline$\rho_{01}$ & & & 0.2756 id & & $0.1100 \mathrm{id}$ & \\
\hline ICC & 0.3268 & & 0.4397 & & 0.4471 & \\
\hline $\mathrm{N}$ & 166 id & & 166 id & & 158 id & \\
\hline Observations & 1884 & & 1884 & & 1800 & \\
\hline Marginal $\mathrm{R}^{2} /$ Conditional $\mathrm{R}^{2}$ & $0.185 / 0.45$ & & $0.264 / 0.58$ & & $0.263 / 0.59$ & \\
\hline
\end{tabular}

Table S2. Summary of mixed models examining associations between sleep duration and subsequent daytime functioning, controlled for mood. Note that the model predicting rumination does not include a random slope as the model with the random slope had a singular fit (random slope and intercept were perfectly correlated). $\sigma 2$ : residual variance; $\tau 00$ : variance of random intercept; $\tau 11$ : variance of random slope; 01 : correlation between random intercept and slope; ICC: intraclass correlation; $\mathrm{N}$ : number of participants. Marginal R2: variance explained by fixed effects; Conditional R2: variance explained by fixed and random effects. P-values were computed with Satterthwaite's approximation. 